\title{
Terahertz field enhancement via coherent superposition of the pulse sequences after a single optical-rectification crystal
}

\author{
Mohsen Sajadi ${ }^{*}$, Martin Wolf and Tobias Kampfrath \\ Fritz Haber Institute of the Max Planck Society, Faradayweg 4-6, 14195 Berlin, Germany.
}

Terahertz electromagnetic pulses are frequently generated by optical rectification of femtosecond laser pulses. In many cases, the efficiency of this process is known to saturate with increasing intensity of the generation beam because of two-photon absorption. Here, we demonstrate two routes to reduce this effect in $\mathrm{ZnTe}(110)$ crystals and enhance efficiency, namely by (i) recycling the generation pulses and by (ii) splitting each generation pulse into two pulses before pumping the crystal. In both methods, the second pulse arrives $\sim 1 \mathrm{~ns}$ after the first one, sufficiently long for optically generated carriers to relax. Enhancement is achieved by coherently superimposing the two resulting terahertz fields.

Over the past two decades, electromagnetic radiation from the terahertz $(\mathrm{THz})$ frequency window has proven to be a highly useful probe of numerous fundamental excitations such as crystal lattice vibrations (in particular in molecular crystals), ${ }^{1}$ free or weakly bound electrons, ${ }^{2,3}$ spin waves, ${ }^{4}$ and spin currents. ${ }^{5}$ In addition to such linear $\mathrm{THz}$ probing, the availability of intense $\mathrm{THz}$ pulses has recently opened the door to applications like nonlinear $\mathrm{THz}$ spectroscopy, ${ }^{6} \mathrm{THz}$ control over matter and light ${ }^{7,8}$ and large-area imaging. ${ }^{9}$ Table-top sources of strong $\mathrm{THz}$ fields generally rely on frequency conversion of intense femtosecond laser pulses in various media, for example, optical rectification (OR) in nonlinear-optical crystals, large-area photoconductive antennas or, in gases, the mixing of the fundamental optical beam and its second harmonic. ${ }^{10}$ A frequently employed material for OR is the nonlinear-optical crystal $\mathrm{ZnTe}$, because of its relatively large second-order optical nonlinearity, low $\mathrm{THz}$ absorption (absorption coefficient $1.3 \mathrm{~cm}^{-1}$ at $1 \mathrm{THz}$ ) and a relatively large phase-matching coherence length ( $>1 \mathrm{~mm}$ for 1 to $3 \mathrm{THz}$ and $800 \mathrm{~nm}$ ). ${ }^{11}$

One may expect higher OR efficiency in ZnTe by just rising the pump intensity or increasing the thickness of the ZnTe crystal. However, this strategy is severely limited because additional nonlinear optical processes become operative. In particular, two-photon absorption (2PA) of the pump beam results in the loss of pump energy and the generation of free charge carriers that dissipate the $\mathrm{THz}$ radiation through Drude-type or free-carrier absorption (FCA). ${ }^{12}$ Consequently, the generated $\mathrm{THz}$ power saturates when the pump power is increased further. ${ }^{12,13,14,15}$ The use of thicker crystals is also problematic as it increases the 2PA-related FCA of THz radiation. (For example, if the thickness of the $\mathrm{ZnTe}$ crystal is doubled, $\mathrm{THz}$ waves generated in the first half slice of the crystal will undergo additional absorption in the second half slice.)

To keep the impact of 2PA and, thus, FCA on a tolerable level, ZnTe(110) crystals with larger aperture have been used, resulting in reduced pump intensity (power per area) and increased opticalto-THz conversion efficiency. ${ }^{16,17}$ For example, using a ZnTe wafer with a diameter as large as $75 \mathrm{~mm}$ and an 800-nm pump pulse with an energy of $48 \mathrm{~mJ}$, Blanchard et al. achieved THz pulses with $1.5 \mu \mathrm{J}$ energy. Note that these values were obtained in a regime close to saturation, and the photon conversion efficiency of $3 \times 10^{-5}$ was orders of magnitude smaller than that of phase-matched OR in $\mathrm{LiNbO}_{3}{ }^{11,18,19}$ Therefore, schemes to further increase the efficiency of OR in ZnTe and crystals with analog saturation behavior are highly desirable.

*sajadi@fhi-berlin.mpg.de 
In this Letter, we demonstrate two routes toward increasing the efficiency of $\mathrm{THz}$ generation via optical rectification in ZnTe. The recycling (RC) scheme [Fig. 1(a)] is based on passing the pump pulse through the ZnTe crystal twice, thereby increasing the generated $\mathrm{THz}$ energy. The split-beam (SB) scheme [Fig. 1(b)] aims at reducing the saturation of the generated $\mathrm{THz}$ field by splitting the generation pulse in two delayed pulses with half the peak intensity. In both the RC and SB scheme, the second pulse is made to arrive $\sim 1 \mathrm{~ns}$ after the first, such that free electrons and holes created by the first pulse have recombined. The resulting two $\mathrm{THz}$ transients are laterally and temporally separated, but coherent superposition of the $\mathrm{THz}$ fields is achieved through a suitable delay line and a focusing element (Fig. 1). Using the RC and SB scheme, we are able to increase the intensity in the $\mathrm{THz}$ focus by a factor of $\sim 1.7$ and $\sim 2.9$, respectively, as compared to single-pulse excitation in which the whole pump energy (without splitting) traverses the crystal only once. This approach is a cost-efficient alternative to strategies based on increasing the crystal area ${ }^{17}$ or thickness, or using several OR media in series. ${ }^{20}$ In particular, the SB approach is useful for all collinearly pumped crystals showing saturation of THz emission, for example organic crystals. ${ }^{21}$

The experimental implementation of schemes RC and SB is schematically shown in Figs. 1(c) and 1(d), respectively. Both setups are driven by an amplified Ti:sapphire laser system (Coherent Legend Elite Duo) that delivers high-power optical pulses (energy $15 \mathrm{~mJ}$, center wavelength $800 \mathrm{~nm}$, duration $40 \mathrm{fs}$, repetition rate $1 \mathrm{kHz}$, beam diameter $20 \mathrm{~mm}$ ). Part of this power is used for pumping the $\mathrm{ZnTe}(110)$ crystal (aperture diameter $20 \mathrm{~mm}$, thickness $0.5 \mathrm{~mm}$ ). In the RC setup [Fig. 1(c)], the first generated THz field is split off the pump by an indium tin oxide (ITO) dichroic mirror and directed toward a parabolic mirror (effective focal length $50.8 \mathrm{~mm}$ ). The isolated pump pulse is fed back into the ZnTe crystal ("recycling"), and the resulting second $\mathrm{THz}$ pulse is aligned such to propagate parallel to the first $\mathrm{THz}$ pulse toward the parabolic mirror. Coherent superposition in the focus is achieved by balancing the delay between the two THz pulses (through moving mirror M3) and by changing the sign of the second $\mathrm{THz}$ field (through the quarter-wave plate) since the $\mathrm{THz}$ fields generated from different sides of the crystal have opposite sign.

In the SB setup [Fig. 1(d)], the pump pulses are split into two beams of equal power, and they traverse the ZnTe crystal with a time delay. As in the RC setup, ITO dichroic mirrors and a parabolic mirror are used to superimpose both $\mathrm{THz}$ pulses. Again, coherent superposition in the common focus is achieved by balancing the delay between the two THz pulses (through moving the dichroic mirror $\mathrm{ITO}_{2}$ ) and by changing the sign of the second $\mathrm{THz}$ field (through the half-wave plate). The superimposed THz electric fields are detected by electro-optic sampling ${ }^{10}$ in another ZnTe(110) crystal (300 $\mu \mathrm{m}$ thickness) that is placed in the focus of the parabolic mirror. As sampling pulses, we employ a fraction of the seed pulses $(2 \mathrm{~nJ}, 800 \mathrm{~nm}, 8 \mathrm{fs}, 80 \mathrm{MHz})$ of our laser system in connection with boxcar detection.

A crucial parameter in both setups is the delay between the two optical pulses arriving at the $\mathrm{ZnTe}$ crystal for OR [Figs. 1(a) and 1(b)]. To reach maximum enhancement, this delay has to be much larger than the lifetime of the 2PA-induced electrons and holes; otherwise the second $\mathrm{THz}$ field will suffer from additional FCA. ${ }^{12}$ As the instantaneous luminescence intensity scales with the number of electrons and holes, ${ }^{22}$ we determine their lifetime by measuring the temporal evolution of the $\mathrm{ZnTe}$ photoluminescence yield following excitation with a femtosecond pump pulse. For this purpose, we employ the broadband fluorescence upconversion setup detailed in Ref. ${ }^{23}$. Figure 2 shows the luminescence intensity as a function of time, and we observe a fast rise on a picosecond time scale, and after $\sim 1 \mathrm{~ns}$, the luminescence and, thus, number of free charge carriers have relaxed to $\sim 3 \%$ of their peak value. The emitted luminescence is centered at $\sim 560 \mathrm{~nm}$, consistent with the $2.26-\mathrm{eV}$ bandgap energy of ZnTe. ${ }^{24}$ Consequently, to minimize 2PA and FCA, we set the delay between the two pump pulses to about $1 \mathrm{~ns}$ in both setups.

We now turn to the key results and start with Fig. 3 which shows $\mathrm{THz}$ transients obtained using the $\mathrm{RC}$ setup. The thick (black) curve represents the $\mathrm{THz}$ transients generated by the first pulse (incident 
fluence $1 \mathrm{~mJ} \mathrm{~cm}^{-2}$ ) and the second (recycled) generation pulse. They are temporally separated to study their shape individually. Note that the recycled transient has the same structure as the first one, however, with a slightly reduced overall amplitude because the second-pass pump pulse has reduced energy owing to 2PA encountered during the first $\mathrm{ZnTe}$ passage. When the two pulses are superimposed optimally (by varying the delay between them), we obtain a transient [thin (red) curve] whose peak field is about $30 \%$ higher than that of the first-pass transient (that is, single-pulse OR). In other words, our RC setup allows us to enhance the THz peak intensity by a factor of $1.3^{2} \sim 1.7$ with respect to single-pulse OR.

Note that scheme RC is to a certain extent equivalent to doubling the thickness of the ZnTe crystal, but it is less susceptible to $2 \mathrm{PA} / \mathrm{FCA}^{13}$ and possibly more cost-efficient. In addition, our approach enables a fully constructive superposition of the two THz pulses, in contrast to a twice as thick ZnTe crystal where phase mismatch causes the $\mathrm{THz}$ wave generated in the first and second half-slice of the crystal to be out of phase. Simulations (based on the formalism of Ref. ${ }^{25}$ ) indicate that this regime is reached at a $\mathrm{ZnTe}$ thickness of $1 \mathrm{~mm}$ and for the generation pulses used in this work.

Typical THz waveforms obtained from our SB setup are displayed in Fig. 4(a). The dashed curve shows the THz transient obtained in case of single-pulse pumping (incident fluence $1 \mathrm{~mJ} \mathrm{~cm}^{-2}$ ). The thick (black) waveform represents the two (temporally separated) $\mathrm{THz}$ pulses obtained when the generation pulse is split in two pulses of equal energy. When the delay between the two pulses is set to zero, we obtain the thin (red) curve whose peak amplitude is $45 \%$ higher than that of the pulse obtained with single-pulse OR (dashed line). In other words, our SB setup substantially increases the efficiency of OR, and this effect can be studied further by varying the energy of the generation pulse.

Along these lines, Fig. 4(b) displays the peak field strength of the THz transient as obtained by singlepulse and SB pumping as a function of the total incident generation pulse fluence. For single-pulse excitation, we did not increase the generation fluence to more than $1.2 \mathrm{~mJ} \mathrm{~cm}^{-2}$ in order to avoid damage of the ZnTe crystal. Above this value, the $\mathrm{THz}$ field is estimated by extrapolating a biexponential fit to the $\mathrm{THz}$ fields obtained with lower pump fluences [dashed line in Fig. 4(b)]. However, when the laser output is equally distributed over two generation pulses (SB scheme), the crystal can be pumped effectively with twice the maximum fluence used for single-pulse excitation. Figure 4(b) also shows the relative field-amplitude increase obtained when switching from the singlepulse to the SB scheme, and we find an increase up to $70 \%$, corresponding to a factor of $1.7^{2 \sim} 2.9$ in terms of intensity.

Note that scheme BS implies a reduction of the generation-beam peak intensity by a factor of 2 . It is, thus, tantamount to increasing the diameter of generation beam and $\mathrm{ZnTe}$ aperture by a factor of $2^{1 / 2}$. However, as $\mathrm{ZnTe}$ and other nonlinear-optical crystals with large diameters are quite expensive, scheme BS is potentially more cost-efficient.

In conclusion, we have demonstrated two routes to increasing the efficiency of OR in ZnTe by either recycling the generation pulse or by distributing the generation energy over two laser pulses. Coherent superposition of the two resulting $\mathrm{THz}$ pulses yielded a $30 \%$ (70\%) field enhancement in the recycling (split-beam) approach at $1 \mathrm{~mJ} \mathrm{~cm}^{-2}\left(2.4 \mathrm{~mJ} \mathrm{~cm}^{-2}\right)$ pump fluence. The SB scheme can easily be scaled to three paths, resulting in an estimated $150 \%$ enhancement of the THz fields at a pump fluence of $2.4 \mathrm{~mJ}$ $\mathrm{cm}^{-2}$. Further enhancements could be achieved by adopting multi-pass schemes as employed in femtosecond laser amplifiers. ${ }^{26}$ Our approach is useful for all collinearly pumped materials showing saturation of $\mathrm{THz}$ emission, including inorganic semiconductors ${ }^{10}$ and organic crystals. ${ }^{21}$

We thank N. Ernsting for providing the transient-fluorescence spectrograph and S. Mährlein for his help in the initial stage of this work. Funding through priority program SPP 1666 of the German Research Foundation (DFG) is gratefully acknowledged. 

Rades, J. Pharm. Pharmacol. 59 (2), 209 (2007). $543(2011)$.

T. Kampfrath, M. Battiato, P. Maldonado, G. Eilers, J. Nötzold, S. Mährlein, V. Zbarsky, F. Freimuth, Y. Mokrousov, S. Bluegel, M. Wolf, I. Radu, P. M. Oppeneer, and M. Münzenberg, Nat. Nanotechnol. 8 (4), 256 (2013).

6 F. Junginger, B. Mayer, C. Schmidt, O. Schubert, S. Mährlein, A. Leitenstorfer, R. Huber, and A. Pashkin, Phys. Rev. Lett. 109 (14), 147403 (2012).

7 T. Kampfrath, K. Tanaka, and K. A. Nelson, Nat. Photonics 7 (9), 680 (2013).

8 M. C. Hoffmann, in Terahertz Spectroscopy and Imaging, edited by K-E. Peiponen, A. Zeitler, and M. Kuwata-Gonokami (Springer Berlin Heidelberg, 2013), Vol. 171, pp. 355.

9 F. Blanchard, A. Doi, T. Tanaka, and K. Tanaka, in Annual Review of Materials Research, Vol 43, edited by D. R. Clarke (2013), Vol. 43, pp. 237.

10 K. Reimann, Rep. Prog. Phys. 70 (10), 1597 (2007).

11 J. Hebling, K-L. Yeh, M. C. Hoffmann, B. Bartal, and K. A. Nelson, J. Opt. Soc. Am. B: Opt. Phys. 25 (7), B6 (2008).

12 S. M. Harrel, R. L. Milot, J. M. Schleicher, and C. A. Schmuttenmaer, J. Appl. Phys. 107 (3), 033526 (2010).

13 S. Vidal, J. Degert, M. Tondusson, J. Oberle, and E. Freysz, Appl. Phys. Lett. 98 (19), 191103 (2011).

14 X. Chen, S. He, Z. Shen, F. L. Zhao, K. Y. Xu, G. Wang, R. Wang, and N. Dai, J. Appl. Phys. 105 (2), 023106 (2009).

15 V. Y. Gaivoronskii, M. M. Nazarov, D. A. Sapozhnikov, E. V. Shepelyavyi, S. A. Shkel'nyuk, A. P. Shkurinov, and A. Shuvaev, Quantum Electron. 35 (5), 407 (2005).

16 T. Löffler, T. Hahn, M. Thomson, F. Jacob, and H. G. Roskos, Opt. Express 13 (14), $5353(2005)$.

17 F. Blanchard, L. Razzari, H. C. Bandulet, G. Sharma, R. Morandotti, J. C. Kieffer, T. Ozaki, M. Reid, H. F. Tiedje, H. K. Haugen, and F. A. Hegmann, Opt. Express 15 (20), 13212 (2007). 
19 S-W Huang, E. Granados, W. R. Huang, K-H. Hong, L. E. Zapata, and F. X. Kaertner, Opt. Lett. 38 (5), 796 (2013).

20 Y. Gao, M-k Chen, S. Yin, P. Ruffin, C. Brantley, and E. Edwards, J. Appl. Phys. 109 (3), 033108 (2011).

21 C. Ruchert, C. Vicario, and C. P. Hauri, Opt. Lett. 37 (5), 899 (2012).

22 H. Wang, K. S. Wong, B. A. Foreman, Z. Y. Yang, and G. K. L. Wong, J. Appl. Phys. 83 (9), 4773 (1998).

23 M. Sajadi, M. Quick, and N. P. Ernsting, Appl. Phys. Lett. 103 (17), 173514 (2013).

24 C. M. Wolfe, N. Holonyak, and G. E. Stillman, Physical Properties of Semiconductors. (Prentice-Hall International, 1989).

25 R. A. Kaindl, F. Eickemeyer, M. Woerner, and T. Elsaesser, Appl. Phys. Lett. 75 (8), 1060 (1999).

26 J. P. Chambaret, C. LeBlanc, G. Cheriaux, P. Curley, G. Darpentigny, P. Rousseau, G. Hamoniaux, A. Antonetti, and F. Salin, Opt. Lett. 21 (23), 1921 (1996). 


\section{Figure captions}

Fig. 1. Schematics of (a) the recycling (RC) and (b) the split-beam (SB) approaches for enhancing the efficiency of $\mathrm{THz}$ generation through $\mathrm{OR}$. In the $\mathrm{RC}$ scheme [(a)], the femtosecond generation pulse is recycled in a second pass, resulting in two $\mathrm{THz}$ pulses that are coherently superimposed by means of a focusing element and a properly chosen delay. In the SB scheme [(b)], saturation effects are reduced by distributing the total pump energy over two delayed pulses, resulting in decreased pump intensity and lower loss of $\mathrm{THz}$ power through 2PA-based FCA. (c) Experimental implementation of scheme RC and (d) scheme SB. Abbreviations: M1-M3: plane silver mirrors, ITO: indium tin oxide dichroic beam splitter for THz and optical radiation, Si-W: silicon wafer, PM: $90^{\circ}$ off-axis parabolic mirror, $\lambda / 2$ : halfwave plate, $\lambda / 4$ : quarter-wave plate. In both setups, the time delay between two sequential THz generation cycles is $\sim 1 \mathrm{~ns}$. In the SB scheme [(d)], the angle between M1, M2 and M3 is slightly less than $90^{\circ}$ to ensure equal delays in both arms.

Fig. 2 Temporal evolution of the photoluminescence intensity of the ZnTe crystal upon excitation with a 40 -fs laser pulse (wavelength of $800 \mathrm{~nm}$, incident fluence $0.35 \mathrm{~mJ} \mathrm{~cm}^{-2}$ ). The photoluminescence is centered at $\sim 560 \mathrm{~nm}$. Following excitation, it rises (see inset) with a time constant of $\tau_{\text {rise }}=2.12$ ps and subsequently decays biexponentially with time constants of $\tau_{1}=50$ ps (relative weight $52 \%$ ) and $\tau_{2}=275$ ps (relative weight $48 \%$ ).

Fig. 3 Transient THz electric fields as obtained using the recycling (RC) scheme [see Fig. 1(a)]. The right- and left-hand pulses of the thick (black) curve show the $\mathrm{THz}$ transients resulting from the first and second (recycling) passes of the generation pulse (incident fluence $0.5 \mathrm{~mJ} \mathrm{~cm}^{-2}$ ). To see both pulses separately, a delay was introduced such that the recycled field arrives 5 ps before the field arising from the first pass. Once both transients are superimposed optimally, the thin (red) signal curve is obtained whose amplitude is $30 \%$ larger than that resulting from a single pass (right-hand pulse of thick curve).

Fig. 4 (a) THz fields (solid lines) obtained using the split-beam (SB) scheme [see Fig. 1(b)]. The dashed curve shows the $\mathrm{THz}$ transient resulting from single-pulse pumping (incident fluence $1 \mathrm{~mJ} \mathrm{~cm}^{-2}$ ). The thick (black) waveform represents the two (temporally separated) $\mathrm{THz}$ pulses when the generation pulse of $1 \mathrm{~mJ} \mathrm{~cm}^{-2}$ fluence is split in two pulses of equal energy. Once the delay between the two pulses is set to zero, the resulting field has a $45 \%$ higher field amplitude than that from single-pulse OR (dashed line). (b) Peak field strength of the THz transient as obtained by single-pulse ( $\bullet$ ) and SB pumping $(\bullet)$ versus total incident generation pulse fluence. Solid blue and red lines are guides to the eyes. The cyan curve displays the relative enhancement of the THz amplitude of the SB to single-pulse scheme. The dashed red line is an extrapolation of the single-pass curve to higher energies based on a biexponential fit. 